\title{
Can Neutrophil-To-Lymphocyte Ratio (NIr) be a simple predictive marker for differentiating gastric intestinal metaplasia and gastric atrophy from non-atrophic non-metaplastic chronic gastritis?
}

\author{
Ufuk Kutluana ${ }^{1, *}$; Ecem Kutluana²; Ayse Kilciler ${ }^{3}$ \\ ${ }^{1}$ Assistant Professor, Pamukkale University Faculty of Medicine, Internal Medicine and Gastroenterology Department, Turkey. \\ ${ }^{2}$ Student, Yeditepe University Faculty of Pharmacy, Turkey. \\ ${ }^{3}$ Professor, Medical Park Bursa Hospital, Internal Medicine and Gastroenterology Department, Turkey.
}

\section{*Corresponding Author: Ufuk Kutluana}

Pamukkale University Medicine Faculty Internal Medicine and Gastroenterology Department. Kinikli Campus Denizli 20070 Turkey.

Tel: +90-5055024770; Email: drufukkana@yahoo.com

Received: Aug 13, 2021

Accepted: Sep 14, 2021

Published: Sep 17, 2021

Archived: www.jjgastro.com

Copyright: (C) Kutluana U (2021).

\section{Abstract}

Background: Gastric Intestinal Metaplasia (GIM) and Gastric Atrophy (GA) are pre-neoplastic lesions that can lead to gastric cancer. Nowadays, there are no recognized good biomarkers of GIM and GA. The neutrophil-to-lymphocyte ratio (NLR) is an economical, effective, and repetitive indicator of inflammation. We aimed to comparatively evaluate Red Cell Distribution Width (RDW) and the NLR.

Methods: 88 patients with GIM and 48 patients with GA and 64 patients with non-atrophic-non-metaplastic gastritis were included in the study. NLR and RDW levels were measured in patients and controls.

Results: NLR levels were significantly higher in patients with GIM than in controls $(p<0.05)$. NLR level was correlated positively with presence of GIM ( $p<0.05)$, H.pylori presence in GIM and GA $(p<0.05)$, and menopause $(p<0.05)$. A multiple logistic regression analysis showed the GIM was predictor for elevated NLR $(p<0.05)$. According to the ROC curve analysis, the best cut-off NLR value to differentiate between patients with GIM from GA and/or controls was $>2.92$ ( $p<0.05)$. In this study, we mainly found a significant association between GIM and NLR.

Conclusions: NLR is significantly higher in patients with GIM. NLR can be an independent determinant factor for GIM.

Keywords: intestinal metaplasia; gastric atrophy; biomarker. 
Citation: Kutluana U, Kutluana E, Kilciler A. Can Neutrophil-To-Lymphocyte Ratio (NIr) be a simple predictive marker for differentiating gastric intestinal metaplasia and gastric atrophy from non-atrophic non-metaplastic chronic gastritis?. Japanese J Gastroenterol Res. 2021; 1(4): 1018.

\section{Introduction}

Gastric Cancer (GC) is the fifth most common malignancy and third leading cause of death from cancer worldwide [1]. It is important to determine at early stages for high-risk group of GC for early diagnosis, management, and effective surveillance. Gastric Intestinal Metaplasia (GIM) and Gastric Atrophy (GA) are pre-neoplastic lesions that can lead to GC. GA is a characterized by the death of the parietal cells and the loss of gastric glandular epithelium. GIM is histologically defined as the replacement of glandular and/or foveolar epithelium by intestinal epithelium and is characterized by its morphological similarity to enterocytes. The most common cause of GIM and GA is Helicobacter pylori (H pylori) gastritis [2]. Both of GIM and AG are intimately associated with immune activation and localized or systemic inflammation [3-6]. The diagnosis of GIM and GA usually based on endoscopic features and histopathological findings. Sometimes it is difficult to make the diagnoses of GA and GIM [7]. The combined measurement of serum pepsinogen I and II (sPGI, sPGII) with serum gastrin-17 (sG17) and GastroPanel have been been proposed as a biomarker of GA [8]. Unfortunately these biomarkers are not are not easily accessible tests everywhere. In addition, nowadays there is no reliable marker of GIM.

Recently, Neutrophil-to-Lymphocyte Ratio (NLR), calculated as a ratio of neutrophil to lymphocyte in peripheral blood, is regarded as a readily available indicator for the severity and extension of systemic inflammation [9-12]. The Red Cell Distribution Width (RDW) is one of the standard parameters which are measured in the complete blood count and it is an indicator of the anisocytosis in the erythrocytary which circulates in the blood. Recently it was shown that elevated RDW levels related with generally increased progressive inflammation, increase in the cytokines and the diseases such as cardiovascular disease, rheumatoid arthritis and inflammatory bowel disease [13-16].

To date no studies have investigated whether both NLR and RDW play a role in the progression of GA and GIM. Thus, the purpose of this study was the first to comparatively evaluate the changes and relationship between NLR and RDW levels in patients with GIM and GA and non-atrophic and non-metaplastic chronic gastritis.

\section{Materials and methods}

A total of 200 prospective and consecutive patients, consistent with the study criteria, who applied to our center, gastroenterology polyclinic, during the period between January 2019 and Dec 2019 and have been included in the study after having obtained their consents. Patients were split into three groups as Gastric intestinal Metaplasia Group (GIMG), Gastric Atrophy Group (GAG) and Control Group (CG). Each group, matched with other for sex, age, menopause, current/past smoking status, and family history of GC. Eighty-eight patients with GIM and fourty-eight patients with GA and CG consisted of sixtyfour patients with nonatrophic gastritis who had no intestinal metaplasia were enrolled to the study. In this study, population characteristics were consist of sex, age, current/past smoking status, menapause, H. pylori infection and family history of GC. The exclusion criteria were as follows: 1, previous H.pylori eradication; 2, intake of antibiotics, nonsteroidal antiinflammatory drugs, statins, proton pump inhibitors, or H2-receptor blockers, antiagregant and anticoagulant in the previous 2 months; 3 , who had been receiving any medications affecting the number of leukocytes; 4, history or presence of cardiovascular disease; 5, diabetes mellitus; 6 , immune-mediated and chronic inflammatory disorders; 7, active infection; 8 , evidence of liver and renal disease; 9, chronic obstructive lung disease; 10, pulmonary hypertension; 11 , malignancy and cytotoxic medicines; 12 , gastric and duodenal ulcer; 13, gastrointestinal hemorrhage; 14 , pregnancy 15 , anemia, vitamin B12, folate deficiency or any hematological disorder; 16 , blood transfusion made in the last three months; 17, Thromboembolic disease; 18, receiving iron deficiency, vitamin B12, folate treatment with their histories and patients who refused the protocol's agreement.

\section{Histopathologic evaluation}

Mucosal samples of all patients were stained with hematoxylin and eosin. Histopathologic parameters were determined according to updated Sydney system [17]. Hematoxylin-eosinstained sections were evaluated for the presence of intestinal metaplasia, gastric atrophy, nonatrophic gastritis (with no intestinal metaplasia) and $\mathrm{H}$. pylori by updated Sydney system.

\section{Assessment of H. pylori Infection}

H. pylori infection was assessed by both histopathologic examination and local rapid urease test. Subjects were considered $\mathrm{H}$. pylori positive to be positive for $\mathrm{H}$. pylori infection if the bacteria were histopathologically detected and/or the local rapid urease test was positive.

\section{Blood samples and biochemical analyses}

The blood samples were obtained from the patients within $12 \mathrm{~h}$ of fasting. The blood samples were collected in contain ethylene diamine tetra acetic acid (EDTA) anticoagulant. Complete blood cell counts and RDW were studied using Mindray BC-6800 (Shenzen Mindray Bio-Medical Electronics Co., Ltd, China). NLR was calculated as a ratio of neutrophil-to lymphocyte in peripheral blood.

The present study was carried out according to the ethics guidelines of the Declaration of Helsinki on biomedical research involving human participants. All patients provided their written informed consent before being involved in the study. The approval of the ethics board was taken for study (number:180-08-14/19/06/2019).

\section{Statistical analysis}

Data were examined by SPSS 23.0 software. Kolmogorov Smirnov and Shapiro-Wilks test were used to evaluate the distribution pattern of the variables in the study. Differences between numeric variables of groups were calculated by analysis of variance (ANOVA) test with post hoc Scheffe method. Chi-square test was used for comparison of categorical variables. A correlation between the NLR levels and variables was assessed by using the Pearson's or Spearman's tests according to the distribution pattern of the variables. The sensitivity and specificity of NLR levels in predicting GIM were calculated using an optimal cut-off point on the Receiver Operating Characteristic (ROC) curve analysis with a $95 \%$ confidence interval. According to the 
results, elevated NLR levels were determined. Stepwise multiple logistic regression was used, considering a normal/elevated NLR levels as the independent variables including GIM, GA and non-atrophic non-metaplastic chronic gastritis. $p<0.05$ was considered to be statistically significant. The Spearman's test and stepwise multiple logistic regression analysis were used for the relationship between normal/elevated NLR levels and the $\mathrm{H}$. pylori status of the histological study groups.

\section{Results}

The characteristics of the GIMG, GAG and CG are shown in (Table 1). There were no significant differences in age, sex, menopause, smoking status, history of GC in family and H.pylori positivity among three groups. In this study, $60.2 \%$ (55/88) of cases with GIM were accompanied by GA. We had no patients with GC in this study. NLR, RDW levels of the groups are shown in Table 2. Serum NLR levels were significantly higher in GIMG than in controls $(2.50 \pm 0.20$ versus $1.87 \pm 0.13, p=0.031)$ (Table 2). NLR level was higher in GIMG than in GAG (2.50 \pm 0.20 versus $2.13 \pm 0.15)$, but this difference did not reveal statistical significance $(p=0.355)$. There was no significant difference in NLR levels in GAG and controls ( $2.13 \pm 0.15$ versus $1.87 \pm 0.13$, $p=0.639)$. There was no significant difference in RDW levels among GIMG, GAG and CG $(16.11 \pm 2.72,16.23 \pm 2.32,15.17 \pm$ 2.27 respectively $\mathrm{p}=0.069$ ).
The associated parameters of NLR level are shown in (Table 3). When Pearson's or Spearman's correlation tests were used, NLR level was correlated positively with type of study groups $(p=0.005)$, presence of GIM $(p=0.008), H$.pylori presence in GIMG ( $p=0.011)$, H.pylori presence in GAG $(p=0.024)$ and presence of menopause $(p=0.031)$

ROC curve analysis revealed that (although the sensitivity was not high), NLR level $>2.92$ was the best cut-off value to differentiate between patients with GIM from GA and/or nonatrophic non-metaplastic chronic gastritis (sensitivity, 30.70\%; specificity, 85.70\%; +likelihood ratio [LR], 2.14;- LR, 0.80; 95\% confidence interval [Cl]: 0.52-0.68; standard error [SE], 0.040; $p=0.014$ ) (Figure 1). The area under the ROC curve (AUC) was 0.619. In GIMG, $32.2 \%$ of the subjects (29/88) had NLR greater than 2.92 . This prevalence was $22.9 \%$ in GAG (11/48) and $12.5 \%$ in CG (8/64). There was significant difference between groups ( $\mathrm{p}=0.019$ ).

Results from the stepwise multiple logistic regression analysis are shown in (Table 4). According to results of analyses, solely the presence of gastric intestinal metaplasia (odds ratio,3.187; 95\% confidence interval, 1.454-6.985; $p=0,004$ ) was predictor for elevated NLR. None of the other variables included in the analysis showed a significant association.

Table 1: Population characteristics in controls and patients with gastric intestinal metaplasia and atrophic gastritis.

\begin{tabular}{|l|c|c|c|c|}
\hline \multicolumn{1}{|c|}{ Parameters } & Gastric intestinal metaplasia group & Gastric atrophy group & Controls & P \\
\hline Age (years) & $51.18 \pm 15.23$ & $46.54 \pm 15.85$ & $53.15 \pm 14.14$ & NS \\
\hline Sex (female/male)(\%) & $55.3 / 44.3$ & $58.3 / 41.7$ & $65.6 / 34.4$ & NS \\
\hline Menopause (\%) & 20.5 & 16.7 & 15.6 & NS \\
\hline Current/past smoker (\%) & 5.7 & 6.9 & 3.1 & NS \\
\hline H.pylori positivity* (\%) & 65.9 & 83.3 & 78.1 & NS \\
\hline History of gastric carcinoma in family (\%) & 4.5 & 10.4 & 3.1 & NS \\
\hline
\end{tabular}

Subjects were considered $\mathrm{H}$. pylori positive to be positive for $\mathrm{H}$. pylori infection if the bacteria were histopathologically detected and/ or the local rapid urease test was positive. ANOVA and Chi-square test were used. $\mathrm{P}<0.05$ was considered to be statistically significant.

Table 2: The laboratory characteristics in controls and patients with gastric intestinal metaplasia and atrophic gastritis.

\begin{tabular}{|c|c|c|c|c|}
\hline Parameters & Gastric intestinal metaplasia group & Gastric atrophy group & Controls & P \\
\hline NLR & $2.50 \pm 0.20$ & $2.13 \pm 0.15$ & $1.87 \pm 0.13$ & P<0.05 \\
\hline RDW (\%) & $16.11 \pm 2.72$ & $16.23 \pm 2.32$ & $15.17 \pm 2.27$ & NS \\
\hline
\end{tabular}

Results were presented as mean \pm SD or median. NLR; neutrophil-to lymphocyte ratio, RDW; red blood cell distribution width. ANOVA test was used. $\mathrm{P}<0.05$ was considered to be statistically significant.

Table 3: The association of NLR level to various parameters.

\begin{tabular}{|l|c|}
\hline Correlation analysis & $\mathrm{p}$ \\
\hline Parameters & $\mathrm{NS}$ \\
\hline Age & $\mathrm{NS}$ \\
\hline Sex & $\mathrm{P}<0.05$ \\
\hline Type of the study groups & $\mathrm{NS}$ \\
\hline H.pylori positivity & $\mathrm{P}<0.05$ \\
\hline Presence of gastric intestinal metaplasia & \\
\hline
\end{tabular}

\begin{tabular}{|l|c|}
\hline Presence of gastric atrophy & NS \\
\hline H.pylori positivity in gastric intestinal metaplasia & $\mathrm{P}<0.05$ \\
\hline H.pylori positivity in gastric atrophy & $\mathrm{P}<0.05$ \\
\hline H.pylori positivity in non-atrophic non-metaplastic gastritis & $\mathrm{NS}$ \\
\hline Smoking status & $\mathrm{NS}$ \\
\hline Family history of gastric carcinoma & $\mathrm{NS}$ \\
\hline Menopause & $\mathrm{P}<0.05$ \\
\hline
\end{tabular}

NLR; neutrophil-to lymphocyte ratio, H. pylori; Helicobacter pylori. Pearson's or Spearman's test was used accordings to the distribution pattern of the variables. $P<0.05$ was considered to be statistically significant. 


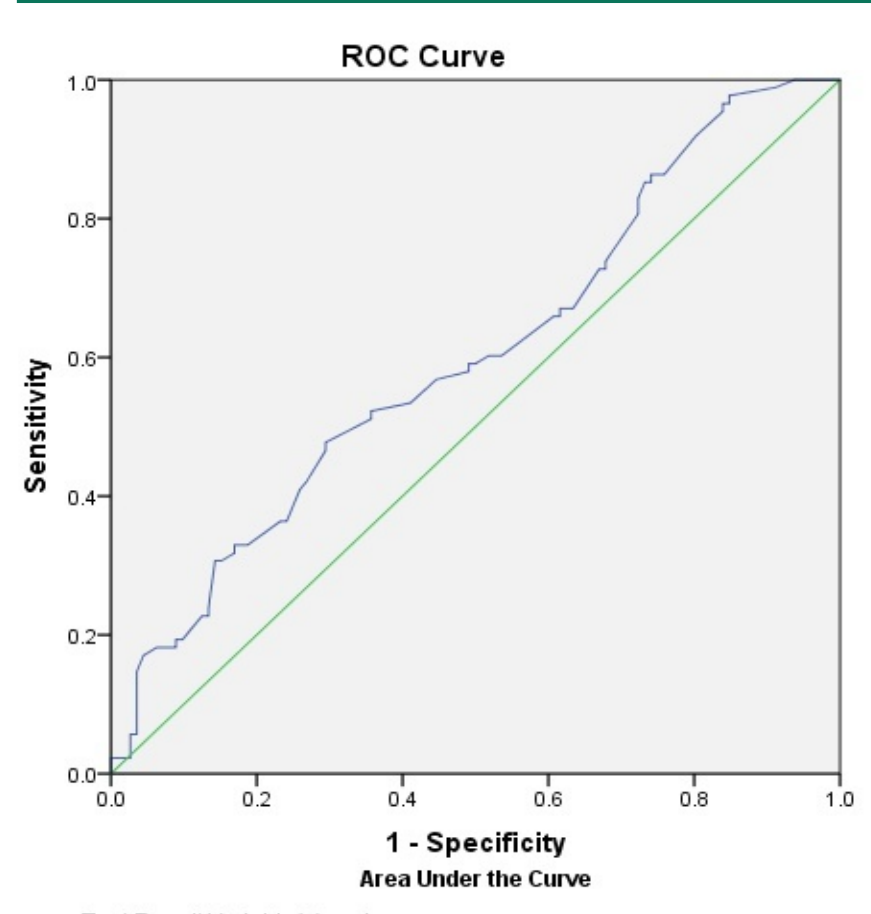

Test Result Variable(s): nlr

\begin{tabular}{|c|c|c|c|c|}
\hline \multirow[b]{2}{*}{ Area } & \multirow[b]{2}{*}{ Std. Error ${ }^{a}$} & \multirow[t]{2}{*}{$\begin{array}{l}\text { Asymptotic } \\
\text { Sig. }^{\text {b }}\end{array}$} & \multicolumn{2}{|c|}{$\begin{array}{c}\text { Asymptotic } 95 \% \text { Confidence } \\
\text { Interval }\end{array}$} \\
\hline & & & Lower Bound & Upper Bound \\
\hline .601 & .040 & .014 & 522 & 680 \\
\hline
\end{tabular}

The test result variable(s): nlr has at least one tie between the positive

actual state group and the negative actual state group. Statistics may be biased.

a. Under the nonparametric assumption

b. Null hypothesis: true area $=0.5$

Table 4: The association of NLR level to various parameters.

\begin{tabular}{|c|c|c|}
\hline \multirow[b]{2}{*}{ Predictors for elevated NLR } & \multicolumn{2}{|c|}{ Logistic regression analysis } \\
\hline & Odds ratio $(95 \% \mathrm{Cl})$ & $\mathrm{p}$ \\
\hline Age & $\begin{array}{l}0.977(0.939 \\
-1.016)\end{array}$ & NS \\
\hline Sex & $\begin{array}{c}0.877(0.259 \\
-2.971)\end{array}$ & NS \\
\hline Menopause & $1.456(0.636-3.337)$ & NS \\
\hline Smoking status & $\begin{array}{l}0.851(0.066- \\
11.041)\end{array}$ & NS \\
\hline Family history of gastric carcinoma & $0.224(0.036-1.418)$ & NS \\
\hline H.pylori positivity & $2.6(0.520-12.993)$ & NS \\
\hline Presence of gastric intestinal metaplasia & $\begin{array}{l}3.187(1.454- \\
6.985)\end{array}$ & $<0,05$ \\
\hline Presence of atrophic gastritis & $\begin{array}{l}2.857(0.675- \\
12.096)\end{array}$ & NS \\
\hline $\begin{array}{l}\text { H.pylori positivity in gastric intestinal meta- } \\
\text { plasia }\end{array}$ & $0.857(0.330-2.229)$ & NS \\
\hline H.pylori positivity in gastric atrophy & $0.395(0.098-1.600)$ & NS \\
\hline $\begin{array}{l}\text { H.pylori positivity in non-atrophic non- } \\
\text { metaplastic gastritis }\end{array}$ & $0.721(0.287-6.071)$ & NS \\
\hline
\end{tabular}

NLR; neutrophil-to lymphocyte ratio, H. pylori; Helicobacter pylori, Stepwise multiple logistic regression was used, considering a normal/elevated ( $\leq 2.92$ versus $>2.92$ ) NLR level (see the text) as the dependent variable and including age (continuous variable), sex (female or male), menopause (presence versus absence), family history of gastric carcinoma (positive versus no history), H. pylori status (positive or negative), gastric intestinal metaplasia (presence versus absence), gastric atrophy (presence versus absence). H.pylori positivity in gastric intestinal metaplasia (presence versus absence), H.pylori positivity in gastric atrophy (presence versus absence), H.pylori positivity in non-atrophic non-metaplastic gastritis (presence versus absence). A p value less than 0.05 was considered statistically significant.

\section{Discussion}

In this study, we mainly found a significant association GIM, and inflammation on the basis of NLR, a simple and reliable indicator of inflammation. We also analyzed RDW formula in this study. We did not find any difference or correlation in RDW levels between the groups. This may be related to the fact that all hematological disorders are within our exclusion criteria, we have limited number of cases and lack of sufficient sensitivity of RDW for GIM and GA without micronutricient deficiency.

The Pelayo Correa cascade delineates a sequence of histological lesions leading to the possible development of GC, beginning with chronic gastritis and evolving into GA, GIM, and finally, cancer [18]. H.pylori is major cause of chronic gastritis. Duodenal ulcer, gastric ulcer and GC are well known disease associated with $\mathrm{H}$. pylori. GIM and GA are most often secondary to chronic long-standing Helicobacter pylori gastritis [19]. It is not clear whether GIM almost always come with GA [20]. In our study, we determined presence of GA in $60.2 \%$ of the patients with GIM. Some studies have demonstrated the following as risk factors for developing GIM: An age of $\geq 61$, the ingestion of spicy food, a history of smoking, male gender, a family history of gastric cancer, the consumption of dairy products, and dietary factors, such as excessive salt consumption, deficient ascorbic acid, and insufficient ingestion of carotene. However, among the risk factors, $\mathrm{H}$. pylori is a major cause of the transdifferentiation into GIM and GA [20]. Molecular pathogenesis of the progression of GIM from H.pylori gastritis has just begun to be understood. The nature of the host immune or inflammatory responses is also important in determining disease consequence after chronic $\mathrm{H}$.pylori gastritis [21]. T helper 1 (Th1) immune response and interferon-gamma (IFN- $\gamma$ ) released from Th1-cells might be important in the progression of GA and intestinal metaplastic formation in a concurrent infection model with Helicobacter felis and helminth. Hence, Th1 predominant inflammatory response to $\mathrm{H}$. pylori may be a key factor of the transdifferentiation to GA and GIM [3]. The nuclear factor kappa $B$ (NF-kB) pathway, which regulates the expression of a wide variety of inflammatory cytokines, is another critical pathway involved in H.pylori induced gastritis and metaplasia [5,6]. Enhanced angiogenesis is another biomechanism that triggers the progression of GIM [22]. Angiogenesis, the sprouting of new capillaries from pre-existing vessels, is a fundamental process accelerating GC progression. Angiogenesis is a complex multistep process regulated by compounding factors. Previous evidence has demonstrated that excessive angiogenesis is significantly correlated with facilitated cell proliferation, the initiation of GIM and progression of GC [22-24].

The increased NLR has been demonstrated in diseases, such as certain malignancies, cardiovascular disease and rheumatoid arthritis [9-13]. NLR is an economical, effective, and repetitive indicator of inflammation [25]. Few studies have shown that there was a correlation between NLR and H.pylori infection $[11,26]$. Aksoy et al. [27] found that RDW and NLR were different in GC patients than in GIM patients. But in their retro- 
spective study, important reasons affecting the complete blood count parameters such as gastrointestinal hemorrhage, anemia, or vitamin B12, folate deficiency are not excluded. Recent researches have reflected that NLR signifies the balance between immunosurveillance (function of anti-tumor immune) and tumor-promoting inflammation (activation of pro-tumor inflammatory pathway). Neutrophils are recognized as the primary source of vascular endothelial growth factor, which serves as an angiogenic mediator involved in tumor angiogenesis, and thusly promoted the development and proliferation of malignancy [28-30]. Zeng et al. showed that increased early angiogenesis in gastric precancerous lesions such as GIM and gastric epithelial dysplasia in rats [22]. To sum up, increased angiogenesis may play a more dominant role for GIM in the gastric carsinogenesis pathway where chronic gastric inflammation turns into GIM or GA. According to the results of our study, distinctive significant elevation of NLR in cases with GIM might be considered as clinic hematologic reflection of Th1, IFN- $\gamma, \mathrm{NF}-\mathrm{KB}$ related immunoreaction and increased angiogenesis.

In present study, H. pylori presence in cases with GIM and GA strongly related with elevated NLR. This observation causes to consider that especially severity of chronic inflammatory process which stems from H.pylori is related to the levels of NLR. Besides, NLR level was correlated positively with menopause in our study. Our this finding is agreement with the previous report in the literature [31].

Another interesting finding in our study was related to NLR cut-off values. ROC curve analysis revealed that although the sensitivity was not high, NLR level $>2.92$ was the best value to differentiate between patients with GIM from GA and/or nonatrophic non-metaplastic chronic gastritis. Even though the sensitivity is low, > 2.92 NLR level with high specificity is promising for GIM. In addition to other serological markers (such as sPGI, sPGII, sG17, GastroPanel and neopterin).

\section{Conclusion}

In conclusion, NLR is significantly higher in patients with GIM. NLR can be an independent determinant factor for GIM. NLR may be a simple, economical and useful marker for detecting especially GIM in the clinical setting and it may help patients not to delay endoscopic screening.

Conflict of interest: We have no any intervening grant or financial support or conflict of interest in this study. The authors of this study declined to declare any competing interests.

\section{References}

1. Jemal A, Bray F, Center MM, Ferlay J, Ward E, Forman D. Global cancer statistics. CA Cancer J Clin 2011; 61: 69-90.

2. Sipponen $\mathrm{P}$, Kekki M, Siurala M. Atrophic chronic gastritis and intestinal metaplasia in gastric carcinoma: comparison with a representative population sample. Cancer. 1983; 52: 1062-8.

3. Fox JG, Beck P, Dangler CA Whary MT, Wang TC, Shi HN et al. Concurrent enteric helminth infection modulates inflammation and gastric immune responses and reduces helicobacter induced gastric atrophy. Nat Med. 2000; 6: 536-542.

4. Devi S, Ansari SA, Vadivelu J Mégraud F, Tenguria S, Ahmed N. Helicobacter pylori Antigen HP0986 (TieA) Interacts with Cultured Gastric Epithelial Cells and Induces IL8 Secretion via NF- ${ }_{K} \mathrm{~B}$ Mediated Pathway. Helicobacter. 2013: 19; 26-36.

5. Rau TT, Rogler A, Frischauf M Jung A, Konturek PC, Dimmler A et al. Methylation-Dependent Activation of CDX1 through NF-kB A
Link from Inflammation to Intestinal Metaplasia in the Human Stomach The American Journal of Pathology. 2012; 181: 487498.

6. Maeda S, Akanuma M, Mitsuno Y Hirata Y, Ogura K, Yoshida H et al. Distinct mechanism of Helicobacter pylori-mediated NF-? ?B activation between gastric cancer cells and monocytic cells. J. Biol. Chem. 2001: 276: 44856-44864.

7. Eshmuratov A, Nah JC, Kim N Lee HS, Lee HE, Lee BH et al. The correlation of endoscopic and histological diagnosis of gastric atrophy. Dig Dis Sci. 2010; 55: 1364-75.

8. Bodger $\mathrm{KI}$, Wyatt JI, Heatley R. Variation in serum pepsinogens with severity and topography of Helicobacter pylori-associated chronic gastritis in dyspeptic patients referred for endoscopy. Helicobacter. 2001; 6: 216-24.

9. Tamhane UU, Aneja S, Montgomery D Rogers EK, Eagle KA, Gurm HS. Association between admission neutrophil to lymphocyte ratio and outcomes in patients with acute coronary syndrome. Am J Cardiol 2008; 102: 653-657.

10. Turkmen K, Guney I, Yerlikaya FH, Tonbul HZ. The relationship between neutrophil-to-lymphocyte ratio and inflammation in end-stage renal disease patients. Ren Fail. 2012; 34: 155-159.

11. Farah R, Farah RK. Association of Neutrophil to Lymphocyte Ratio With Presence and Severity of Gastritis Due to Helicobacter pylori Infection. Journal of Clinical Laboratory Analysis. 2014; 28: 219-223.

12. Sato $\mathrm{Y}$, Gonda K, Harada M, Tanisaka Y, Arai S, Mashimo $\mathrm{Y}$ et al. Increased neutrophil-to-lymphocyte ratio is a novel marker for nutrition, inflammation and chemotherapy outcome in patients with locally advanced and metastatic esophageal squamous cell carcinoma. Biomedical Reports. 2017: 7; 79-84.

13. Nishizaki Y, Yamagami S, Suzuki H, Joki Y, Takahashi S, Sesoko M et al. Red blood cell distribution width as an effective tool for detecting fatal heart failure in super-elderly patients. Intern Med. 2012; 51: 2271-76.

14. Gonzalo CD, Luxan-Delgado B, Rodriguez GS, Garcia MM, Suarez FM, Solano JJ et al. Interleukin 6, soluble tumor necrosis factor receptor I and red blood cell distribution width as biological markers of functional dependence in an elderly population: $A$ translational approach. Cytokine. 2012; 58: 193-8.

15. He Y, Liu C, Zeng Z, Ye W, Lin J, Ou Q. Red blood cell distribution width: a potential laboratory parameter for monitoring inflammation in rheumatoid arthritis. Clin Rheumatol. 2018; 37: 161167.

16. Song CS, Park DI, Yoon MY, Seok HS, Park JH, Kim HJ. et al. Association between red cell distribution width and disease activity in patients with inflammatory bowel disease. Dig Dis Sci. 2012; 57: 1033-8.

17. Dixon MF, Genta RM, Yardley JH, Correa P. Classification and grading of gastritis. The updated Sydney system. International Workshop on the Histopathology of Gastritis. Am J Surg Pathol 1996; 20: 1161-1181.

18. Correa P, Piazuelo MB, Camargo MC. Etiopathogenesis of gastric cancer. Scand J Surg. 2006; 95: 218-24.

19. Asaka M, Sugiyama T, Nobuta A. Atrophic gastritis and intestinal metaplasia in Japan: results of a large multicenter study. Helicobacter. 2001; 6: 294-299.

20. Sepulved AR. Improvement of Gastric Atrophy After Cure of Helicobacter pylori What We Know, What We Don't Know, and Why Care? J Clin Gastroenterol. 2003; 36: 382-384. 
21. Blanchard TG, Czinn SJ. Immunological determinants that may affect the Helicobacter pylori cancer risk. Aliment Pharmacol Ther. 1998; 12: 83-90.

22. Zeng J, Ran YJ, Pan H, You F, Cai T, Liu W et al. Weipixiao attenuate early angiogenesis in rats with gastric precancerous lesions. Complementary and Alternative Medicine. 2018; 18: 250.

23. Zhang $X$, Zheng Z, Shin YK, Kim KY, Rha SY, Noh SH et al. Angiogenic factor thymidine phosphorylase associates with angiogenesis and lymphangiogenesis in the intestinal-type gastric cancer. Pathology. 2014; 46: 316-24.

24. Maeda K, Chung YS, Takatsuka S, Ogawa Y, Onoda N, Sawada $T$ et al. Tumour angiogenesis and tumour cell proliferation as prognostic indicators in gastric carcinoma. Br J Cancer. 1995; 72: 319-23.

25. Azab B, Chainani V, Shah N, Mcginn JT. Neutrophil-lymphocyte ratio as a predictor of major adverse cardiac events among diabetic population: a 4-year follow-up study. Angiology. 2013; 64: 456-465.

26. Kaplan M, Ates I, Yuksel M, Arikan MF, Aydog G, Turhan N et al. The role of the PLR-NLR combination in the prediction of the presence of Helicobacter pylori and its associated complications. Saudi J Gastroenterol 2018; 24: 294-300.

27. Aksoy EK, Kantarcı S, Torgutalp M, Akpınar MY, Sapmaz FP, Yalçın GŞ, et al. The importance of complete blood count parameters in the screening of gastric cancer Gastroenterology Rev 2019;
14: 183-187.

28. Kusumanto YH, Dam WA, Hospers GA, Meijer C, Mulder NH et al. Platelets and granulocytes, in particular the neutrophils, form important compartments for circulating vascular endothelial growth factor. Angiogenesis. 2003; 6: 283-87.

29. Jablonska J, Leschner S, Westphal K, Lienenklaus S, Weiss S. Neutrophils responsive to endogenous IFN-beta regulate tumor angiogenesis and growth in a mouse tumor model. J Clin Invest. 2010; 120: 1151-64.

30. Hofman PM. Pathobiology of the neutrophil-intestinal epithelial cell interaction: role in carcinogenesis. World J Gastroenterol. 2010; 16: 5790-800.

31. Liu W, Huang Z, Tang S, Wei S, Zhang Z. An evaluation of homocysteine, C-reactive protein, lipid levels, neutrophils to lymphocyte ratio in postmenopausal osteopenic women. Gynecol Endocrinol. 2016; 32: 446-8. 